\title{
Enhanced residual mean circulation during the evolution of split type sudden stratospheric warming in observations and model simulations
}

\author{
Sourabh Bal ${ }^{1,4, *}$, Semjon Schimanke ${ }^{2}$, Thomas Spangehl ${ }^{3}$ and Ulrich Cubasch ${ }^{1}$ \\ ${ }^{1}$ Institute for Meteorology, Freie Universitat, Berlin, Germany. \\ ${ }^{2}$ Swedish Meteorological and Hydrological Institute, Norrköping, Sweden. \\ ${ }^{3}$ German Weather Service, Offenbach am Main, Germany. \\ ${ }^{4}$ Department of Physics, Swami Vivekananda Institute of Science and Technology, Kolkata, India. \\ *Corresponding author. e-mail: sourabhbal@gmail.com
}

MS received 10 April 2017; revised 27 November 2017; accepted 12 December 2017; published online 26 June 2018

Residual mean circulation changes during the evolution of sudden stratospheric warming (SSW) are investigated by composite analyses of 76 major warming events identified in a present day simulation performed with a coupled ocean-troposphere-stratosphere model from 299 winters. Their dynamical signatures are compared with the 17 SSW events identified from 35 years of Era-Interim data. The main difference is that, relative frequency of simulated SSW events is smaller than that obtained from reanalysis. SSW events are classified as displacement or split events based on the geopotential field values at $10 \mathrm{hPa}$. The geopotential field values identify 10 and 3 split events in simulation and observation respectively. The model quite accurately simulates some of the dynamical features associated with the major SSW. Residual mean circulation induced by EP-flux divergence, sum of advection and residual forcing are stronger in split events than in displacement type SSW has been confirmed by both simulation and observation. Moreover, the contribution of EP-flux divergence or planetary wave forcing is larger than the contribution of other types of forcing.

Keywords. Split events; stratospheric sudden warming; residual mean circulation.

\section{Introduction}

Sudden stratospheric warming (SSW) was first noted by Scherhag (1952) over Berlin on January 30, 1952. He observed an unusual rise in temperature at $10 \mathrm{hPa}$. Later, Charney and Drazin (1961) demonstrated that during winter when the prevailing winds are steady and westerly, stationary planetary waves, which are mainly induced by the nonuniform orography of the northern hemisphere, can penetrate into the stratosphere. Eddies which develop through different dynamical processes with first and second order zonal harmonics referred to as planetary waves (Matsuno 1971). These vertically growing planetary waves transport heat and momentum from mid-latitudes into the polar stratosphere. Within few days, with the sudden increase in wave amplitude, there is rise in temperature over the pole and the zonal winds slow down or reverse from westerly to easterly. Recently, a study by Butler et al. (2015) suggested numerous definition of SSW and also reported that the frequency of SSW events is sensitive to the exact definition of SSWs. World Meteorological Organization 
(WMO) classified sudden stratospheric warming as major warming, if the polar cap temperature increases by few tens of kelvin within a week. In addition to increase in the polar cap temperature, reversal mean zonal wind from westerly to easterly also takes place. In minor warming, mean zonal winds in the stratosphere weaken, but do not reverse with the increase in zonal temperature of small amplitude. A major sudden stratospheric event which occurs in late winter is termed as final warming. Canadian warming takes place when the Aleutian stratospheric high intensifies and moves poleward. These warmings which often happen in early winter can reverse the meridional temperature gradient and sometimes slightly change the zonal wind direction over the polar cap, nevertheless they do not lead to a breakdown of the cyclonic polar vortex (Labitzke 1981). The amplified planetary waves during sudden stratospheric warming generated are wave number 1 and 2. Wave number 1 of certain amplitude is necessary for a major warming to occur. Wave number 2 is not sufficient to generate major warmings (Charlton and Polvani 2007; Harada et al. 2010). Sudden stratospheric warming events are also classified as Type A and Type B stratospheric warmings (Schoeberl 1978). In Type A warmings, planetary waves of wave number 2 amplifies first two weeks prior to the onset of the warming. Then from the key date, the wave number 1 amplifies with the weakening of wave 2. Type A warming includes major, minor or final warming. In Type B warming, planetary wave number 1 remain amplified for a longer period. Major stratospheric warming is the best example of Type B warming (Harada et al. 2010). Warmings are also classified as vortex displacement and vortex split events. The vortex split and displacement events are categorized based on the synoptic structure. Displacement type is characterized by a clear shift of the polar vortex from the pole. Vortex split events are clearly visible when polar vortex breaks up into two pieces (Charlton and Polvani 2007). Vortex intensification (VI) is the opposite phenomenon of sudden warming. It is characterized by a rapid cooling of the polar cap temperature (Kuroda 2008). The competition between planetary waves and gravity waves to the residual mean circulation associated with different types of sudden warming are studied by Limpasuvan et al. (2012) and Albers and Birner (2014). Recently, Bal et al. (2017) studied the influence of northern hemisphere warming events on the convective activity in the equatorial troposphere using Era-Interim.
They showed that ensemble of all warming events produce vertical EP-flux which strengthens few days before the key date corresponding to the negative divergence in the lower stratosphere. During these warming events, negative temperature change in the equatorial lower stratosphere takes place due to increased Brewer-Dobson circulation. The negative temperature in the upper troposphere invites upwelling from the tropics. It was discussed in the study of Bal et al. (2017) that the number of SSW events in observations are too sparse to analyse displacement and vortex split events in the middle atmosphere circulation. Therefore, in this study, we examine the changes in the zonal-mean flow during both of these types of warming event (displacement and vortex split) through composite analysis. The present research effort evaluates 17 warming events from the 35 years of Era-Interim (1979-2013) and compares with 76 events from 300 model years of present day simulation (PD).

The paper is organized as follows. We describe the datasets from observation and simulation in section 2 . In section 3 , we calculate the key dates of the warming and classify them into displacement and split events. The method to calculate the variables of residual mean circulation are explained in section 4. Section 5 interprets the results. Zonal winds and temperature anomalies are described in section 5.1. The change of different components of residual mean circulation is explained in section 5.2 with conclusions and discussion in section 6 .

\section{Model and data}

In this paper, we consider 300 model years representing present day conditions from the series of experiments presented by Schimanke et al. (2011, 2013). The experiments are performed with the Atmosphere-Ocean General Circulation Model EGMAM that is based on ECHO-G (Legutke et al. 1999), but vertically extended. The atmospheric component (MA-ECHAM4) model has a horizontal resolution of T30 and 39 vertical levels with the extended model top at $0.01 \mathrm{hPa}$ (approximately $80 \mathrm{~km}$ ) which covers the stratosphere and extends to the upper mesosphere. The zonal mean zonal wind structure computed from EGMAM during northern hemisphere winter season is comparable to ERA40 reanalysis. The model captures the observed temperature patterns in the middle atmosphere and tracks the downward propagation of stratospheric vortex anomalies down to the 
troposphere similar to observations. Moreover, EGMAM establishes a reasonable relationship between the meridional heat flux at the troposphere and the temperature variability of the stratospheric polar winter vortex. The coupled ocean part (HOPE-G) has $2.8^{\circ}$ horizontal resolution with equator refinement and 21 vertical levels. The improved vertical resolution of this model failed to produce a quasi-biannual oscillation. It is to be noted that the model produces permanent easterly winds in the lower tropical stratosphere and thus the QBO variability is missing. Even the tropospheric westerly wind at $60^{\circ} \mathrm{N}$ are too weak and the subtropical jet is slightly overestimated in EGMAM. The model depicts the downward advancement of stratospheric polar winter vortex anomalies down to the troposphere comparable to observations (Schimanke et al. 2013). The model slightly underestimates the number of SSWs as westerly winds around $60^{\circ} \mathrm{N}$ at $10 \mathrm{hPa}$ is slightly too strong are elaborated in several studies (Huebener et al. 2007; Spangehl et al. 2010) and shows that EGMAM is within the range of most successful models. A comprehensive description of the model is illustrated in Schimanke et al. (2013). The control experiment of 20th century coined as present day simulation (PD) with constant $\mathrm{CO}_{2}$ of 352.0 ppmv similar to twentieth century condition and radiative forcing approximately $1.9 \mathrm{~W} / \mathrm{m}^{2}$ during 1990 are used (Schimanke et al. 2013).

Zonal wind velocity, meridional velocity, vertical velocity, temperature and geopotential heights are employed for this study. The output from the simulation are compared and further examined considering Era-Interim data of Dee et al. (2011). The Era-Interim are accessible for 31 vertical levels from $1000 \mathrm{hPa}$ to $0.1 \mathrm{hPa}$. More details about the datasets can be found at the European Centre for Medium-Range Weather Forecasts (ECMWF).

\section{Selection and classification of SSW}

We used the algorithm established by Schimanke et al. (2013) which is also used by Bal et al. (2017) to calculate the warming dates from EraInterim. This numerical scheme was developed with a backbone following Charlton and Polvani (2007). Details of the numerical function are explained in Schimanke et al. (2013). The key date for each warming date according to WMO is computed on the day when reversal of zonal wind at $10 \mathrm{hPa}$ takes place (Charlton and Polvani 2007).
The algorithm used in our study, identifies 17 and 76 warming events from reanalysis and model simulation respectively. To distinguish between vortex split and vortex displacement events, we observe the geopotential height field at $10 \mathrm{hPa}$ in polar stereographic projection around the central date. In figure 1, geopotential field at $10 \mathrm{hPa}$ with selected 3-day means before and after the key date are plotted for both simulation and Era-Interim. First two rows from the top represent the displacement and split SSW from Era-Interim. EGMAM reproduces both displacement and split SSW which is indicated in figure 1 (last two rows). There is good agreement between the model and the reanalysis in the geopotential field value at $10 \mathrm{hPa}$ for displacement type events in particular. In split SSW, the exhibited two maxima and two minima of approximately equal magnitude of geopotential field values are more pronounced in Era-Interim than in the model. In EGMAM, the seperation of polar vortex is well separated and high pressure is also developed over the north pole but not separated into poles. Classification of SSW events into displacement and split SSW are evaluated under different criteria (Charlton and Polvani 2007; Torre et al. 2012; Ryoo and Chun 2005). Charlton and Polvani (2007) identified split SSW based on the absolute vorticity on pressure surface as a substitute for Ertel potential vorticity. We observed three split events from geopotential field stereographic plots at $10 \mathrm{hPa}$ from Era-Interim after following the similar definition of Torre et al. (2012). The number of SSW/winter computed by Torre et al. (2012) is 0.64 for ERA-40 which is slightly more than in our study, i.e., 0.50 for Era-Interim. All the three split events identified in our study occurred in January which underestimates the number of split events computed by Torre et al. (2012). This less number of split events identified in our study may be explained due to the fact that SSW classification is calculated from individual geopotential field values for each SSW around central date without any automated scheme. The split events computed by the geopotential heights from Era-Interim lies in 1984/85, 2008/09 and 2012/13 winters. Vortex split events are connected with large planetary wave activity of wave number 2 (Mitchell et al. 2011). The three winters $(1984 / 85,1988 / 89$ and 2008/09) have been identified by Harada et al. (2010) using Japanese 25-yr Reanalysis Project data which infers that the wave 2 contributes more than $60 \%$ compared to the sum of wave 1 and wave 2. Earlier Charlton and Polvani (2007) 
(a) displacement event (ERAINT)

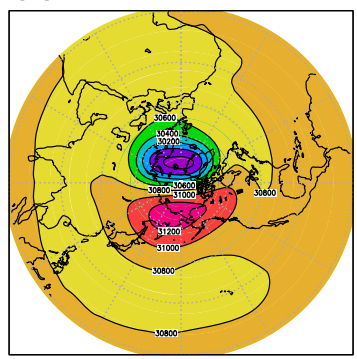

(a) split event (ERAINT)

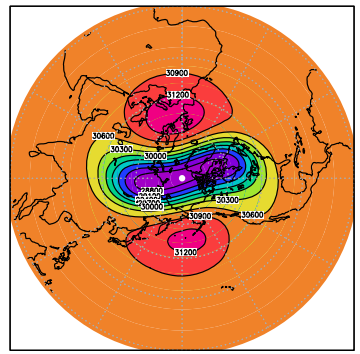

(a) displacement event (PD)

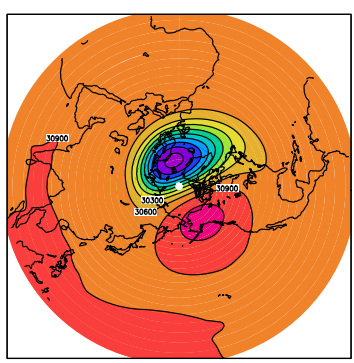

(a) split event (PD)

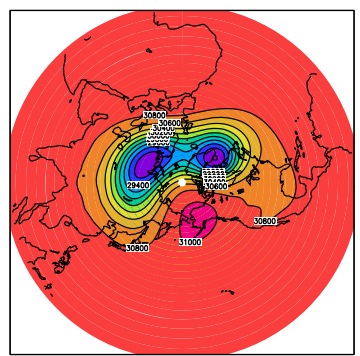

(b) displacement event (ERAINT)

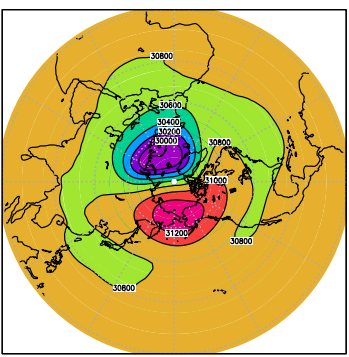

(b) split event (ERAINT)

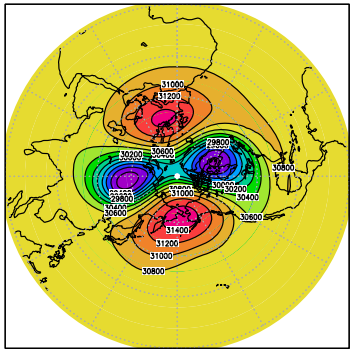

(b) displacement event (PD)

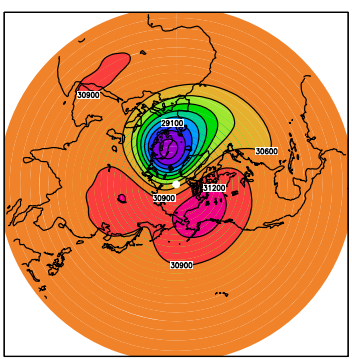

(b) split event (PD)

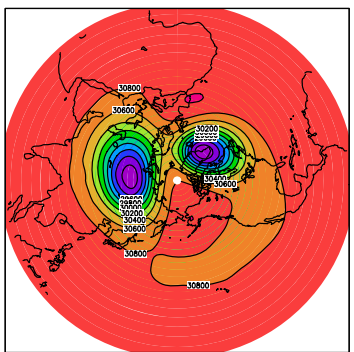

(C) displacement event (ERAINT)

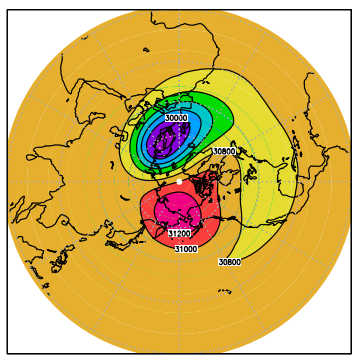

(c) split event (ERAINT)

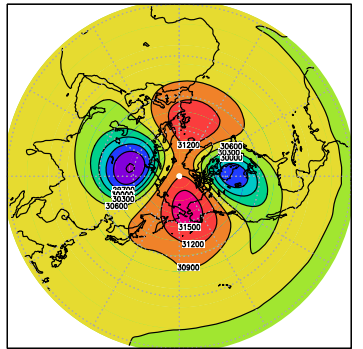

(C) displacement event (PD)

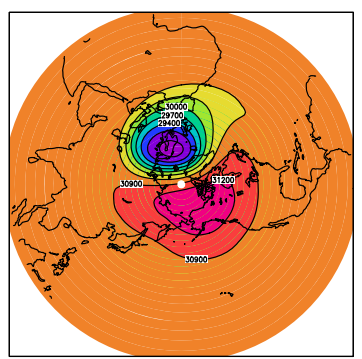

(C) split event (PD)

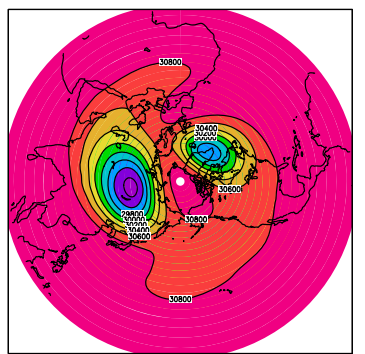

(d) displacement event (ERAINT)

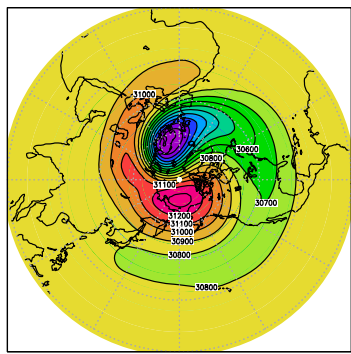

(d) split event (ERAINT)

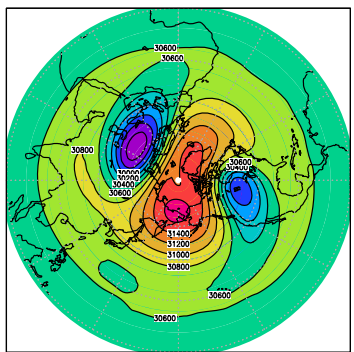

(d) displacement event (PD)

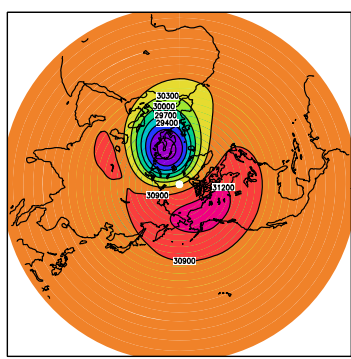

(d) split event (PD)

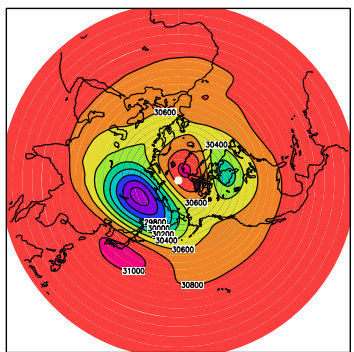

Figure 1. Geopotential field at $10 \mathrm{hPa}(\mathbf{a})$ averaged day $=-6$ to day $=-4$, (b) averaged day $=-3$ to day $=-1$, (c) averaged day $=0$ to day $=+2$, and $(\mathbf{d})$ averaged day $=+3$ to day $=+5$ for displacement and split type SSW in Era-Interim (row 1 and row 2) and in PD (row 3 and row 4) respectively.

had identified 15 split events using NCEP-NCAR and ERA-40 datasets from 1958 to 2002. Comparison of split events with Charlton and Polvani (2007) yields one vortex split event (1984/85) in common with our classification using Era-Interim from 1979 to 2013. Thus, five additional split events (1986/87, 1987/88, 1988/89, 1998/99 and 2000/01) identified by Charlton and Polvani (2007) are not captured in our classification using geopotential values at $10 \mathrm{hPa}$. To examine the efficiency of our numerical scheme, we have compared the stratospheric warming computed from Era-Interim with warming events analyzed by Freie Universitat, Berlin (FUB). The comparison is presented in figure 2(a). It is evident from figure 2(a), that the ratio of the SSW computed by FUB and reanalysis do not agree with all winters, but matches well with maximum winter years. Table 1 shows the central date and duration of 17 SSW events from reanalysis. Vortex split SSWs are indicated with asterisk. 

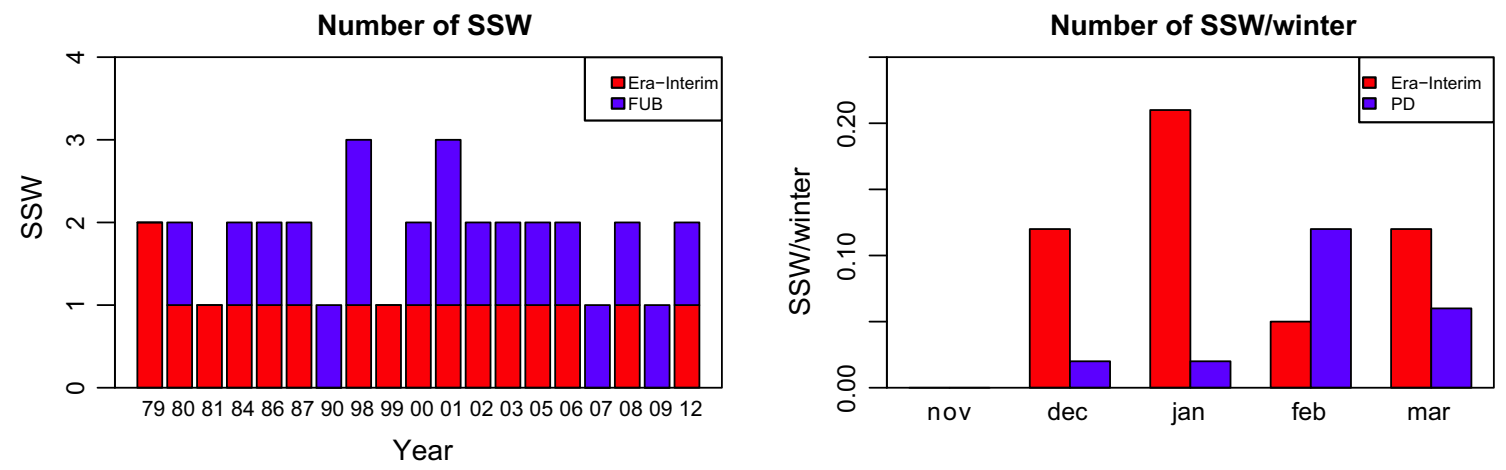

Figure 2. Left panel: Comparison of computed SSW number from Era-Interim (Schimanke et al. 2013) with Freie Universitat, Berlin (FUB) (Labitzke and Naujokat 2000). Right panel: Monthly frequency of all SSW events for PD and Era-Interim.

Table 1. SSW key dates from ERAInterim using algorithm of (Schimanke et al. 2013).

\begin{tabular}{lc}
\hline SSW keydate & $\begin{array}{c}\text { Duration } \\
\text { (in days) }\end{array}$ \\
\hline $1980-03-01$ & 2 \\
$1980-03-17$ & 9 \\
$1981-03-04$ & 1 \\
$1981-12-04$ & 2 \\
$1985-01-01^{*}$ & 6 \\
$1987-01-23$ & 33 \\
$1987-12-08$ & 9 \\
$1998-12-15$ & 7 \\
$2000-03-20$ & 3 \\
$2001-02-11$ & 14 \\
$2001-12-30$ & 4 \\
$2003-01-18$ & 1 \\
$2004-01-05$ & 10 \\
$2006-01-21$ & 26 \\
$2007-02-24$ & 4 \\
$2009-01-24^{*}$ & 30 \\
$2013-01-06^{*}$ & 22 \\
\hline
\end{tabular}

*Denotes vortex split events computed using geopotential heights in this present study.

It is indicated in table 1 that there are six warming events from observations, which lasts for 10 days or more. Out of these six enduring events, two events are identified as split events. There are 18 events counted by FUB in the same 35-year span and the present algorithm computes one event less. Thus the development of the present algorithm is an advancement in identifying the events. Monthly frequency of the SSW events are also presented in figure 2(b) where it shows the comparison of number of SSW/winter for both Era-Interim and model. The algorithm did not find any warming events in November for both the datasets. The number of SSW/winter for each month is underestimated in PD in contrast to the reanalysis except for February. Thus the model computes significantly less SSW. We use geopotential height values to classify each warming events into displacement and split type major stratospheric warming events. Following these criteria, there are three split events from 17 SSWs in Era-Interim and 10 split SSW out of 76 major warming events in PD.

\section{Methodology}

Daily vertical and horizontal components of the residual circulation, EP-flux and its divergence have been calculated based on the following equations from Andrews et al. (1987).

$$
\begin{aligned}
\frac{\partial \bar{u}}{\partial t}= & f \bar{v}^{*}-\frac{\bar{v}^{*}}{a \cos \phi} \frac{\partial}{\partial \phi}(\bar{u} \cos \phi)-\bar{\omega}^{*} \bar{u}_{z} \\
& +\frac{1}{\rho_{0} a \cos \phi} \nabla \cdot \mathbf{F}+\bar{X} .
\end{aligned}
$$

In equation (1), zonal-mean zonal momentum tendency is balanced by $f \bar{v}^{*}$ (coriolis force due to the Brewer-Dobson (B-D) circulation), $\frac{\bar{v}^{*}}{a \cos \phi} \frac{\partial}{\partial \phi}(\bar{u} \cos \phi)-\bar{\omega}^{*} \bar{u}_{z}$ (advection of the zonalmean zonal momentum by the B-D circulation), $\frac{1}{\rho_{0} a \cos \phi} \boldsymbol{\nabla} \cdot \mathbf{F}$ (EP-flux or planetary wave forcing) and the residual forcing expressed as $\bar{X}$. Here $v^{*}$ and $\omega^{*}$ represent meridional and vertical components of the residual mean circulation defined in equations (2) and (3). $u, v$ and $\omega$ symbolize zonal, meridional and the vertical wind, respectively. $\boldsymbol{\nabla} \cdot \mathbf{F}$ is the divergence of the EP-flux vector which signifies divergence of the eddy heat and eddy momentum fluxes. The components of the EP-flux vector $\mathbf{F}$ are presented in equations (4 and 5). 


$$
\begin{aligned}
& \bar{v}^{*}=\bar{v}-\frac{1}{\rho_{0}} \frac{\partial}{\partial z}\left(\rho_{0} \frac{\overline{v^{\prime}} \bar{\theta}^{\prime}}{\overline{\theta_{z}}}\right) \\
& \bar{\omega}^{*}=\bar{\omega}+\frac{1}{a \cos \phi} \frac{\partial}{\partial \phi}\left(\cos \phi \frac{\overline{v^{\prime} \bar{\theta}^{\prime}}}{\overline{\theta_{z}^{\prime}}}\right) \\
& \mathbf{F}^{(\phi)}=\rho_{0} a \cos \phi\left(\bar{u}_{z} \frac{\overline{v^{\prime}} \bar{\theta}^{\prime}}{\overline{\theta_{z}}}-\overline{v^{\prime}} \overline{u^{\prime}}\right) \\
& \mathbf{F}^{(z)} \\
& =\rho_{0} a \cos \phi\left[\left(f-\frac{1}{a \cos \phi} \frac{\partial}{\partial \phi}(\bar{u} \cos \phi)\right) \frac{\overline{v^{\prime}} \bar{\theta}^{\prime}}{\overline{\theta_{z}}}-\overline{\omega^{\prime}} \bar{u}^{\prime}\right]
\end{aligned}
$$

In this work, anomalies are calculated using daily climatology. Anomalies for each field of interest are calculated using deviation from its daily climatology. It has been discussed that internal variability like QBO contaminates SSW signal (Baldwin and Dunkerton 2001; Taguchi 2011; Gómez-Escolar et al. 2014). So, we employed different filtering techniques (low pass, high pass, band pass) to remove the internal variability. The anomalies computed using different filters do not show any significant difference with daily climatology.

\section{Results}

\subsection{Zonal mean temperature and zonal mean zonal winds during stratospheric warming}

Figure 3 compares the zonal wind between $50^{\circ}$ and $70^{\circ} \mathrm{N}$ (column 1) and zonal mean temperature (column 2) averaged over $70^{\circ}-90^{\circ} \mathrm{N}$ associated with Era-Interim (top) and model simulation (bottom). Dots in each panel of the remaining figures indicate regions greater than $95 \%$ significance by the Student- $t$ criterion. The composite of zonal wind and zonal temperature anomalies in the polar region during the 90-day period centered around the key dates are shown in this plot. Here day $=0$ is defined as the key date of the SSW events. The vertical axis represents the height and the horizontal axis shows the time in days before and after the SSW events from the key date. For the time evolution of SSW, the anomalous zonal wind at $10 \mathrm{hPa}$ (figure $3 \mathrm{a}, \mathrm{c}$ ) is stronger until around day = -10 , weakens very quickly from then onwards and becomes easterly with a value greater than $8 \mathrm{~ms}^{-1}$ on day $=0$ and remains negative until around 3 weeks after the events. This anomalous zonal wind pattern is observed for both reanalysis data and model output. The retardation of the zonal wind and transition to easterly takes place earlier in the model than in Era-Interim. During a major SSW event, the stratospheric circulation changes dramatically and the westerly zonal winds that prevail at $10 \mathrm{hPa}$ become the easterly winds. In addition to the changes in zonal wind, anomalous zonal temperature is also observed in the polar region. The anomalous stratospheric zonal temperature in the $70-90^{\circ} \mathrm{N}$ region is cooler before the day $=-2$ in Era-Interim (figure $3 \mathrm{~b}$ ), but the stratospheric zonal temperature at $10 \mathrm{hPa}$ begins to increase 3 weeks prior to central date in PD (figure $3 \mathrm{~d}$ ). The temperature reaches $10^{\circ}$ and $12^{\circ} \mathrm{C}$ for Era-Interim and PD, respectively at $10 \mathrm{hPa}$ level on day $=0$ and are really close to each other considering the normal state around $-40^{\circ}$ to $-60^{\circ} \mathrm{C}$. After the final day of the warming, the temperature begins to cool, but it remains warmer specially in the lower stratosphere and upper troposphere until a month in both the datasets. The temperature at $10 \mathrm{hPa}$ becomes cooler and it propagates downward from day $=+20$ in Era-Interim and 5 days later in PD. The overall temperature change well captures the quasi-periodic time evolution of the polar-night jet oscillation (PJO) as reported by Kodera (2006). Such changes in temperature and zonal wind should be closely related to the wave propagation from troposphere.

\subsection{Residual mean circulation during stratospheric warming}

The EP-flux divergence is the primary retarding forcing of the zonal wind in the $\mathrm{NH}$ winter (Monier and Weare 2011). EP-flux divergence is the resultant of the horizontal divergence of the meridional eddy momentum flux and the vertical divergence of the meridional eddy heat flux. It has been shown by Monier and Weare (2011) that eddy momentum flux and eddy heat flux act together resulting in a westward body force that decelerate the polar vortex in the NH wintertime. In addition, the presence of a greater land area and topography distribution in the NH results in a stronger contribution from stratospheric process which are forced by topography and land-sea heating contrasts.

Figure 4 shows composite of EP-flux divergence with flux vectors, zonal wind tendency, the advection of zonal-mean zonal momentum by the B-D circulation defined as the sum of advection and residual forcing with residual mean circulation direction for displacement and split type SSW respectively. The $\mathrm{x}$-axis denotes 15 days before and after the final warming and the vertical 
(a)

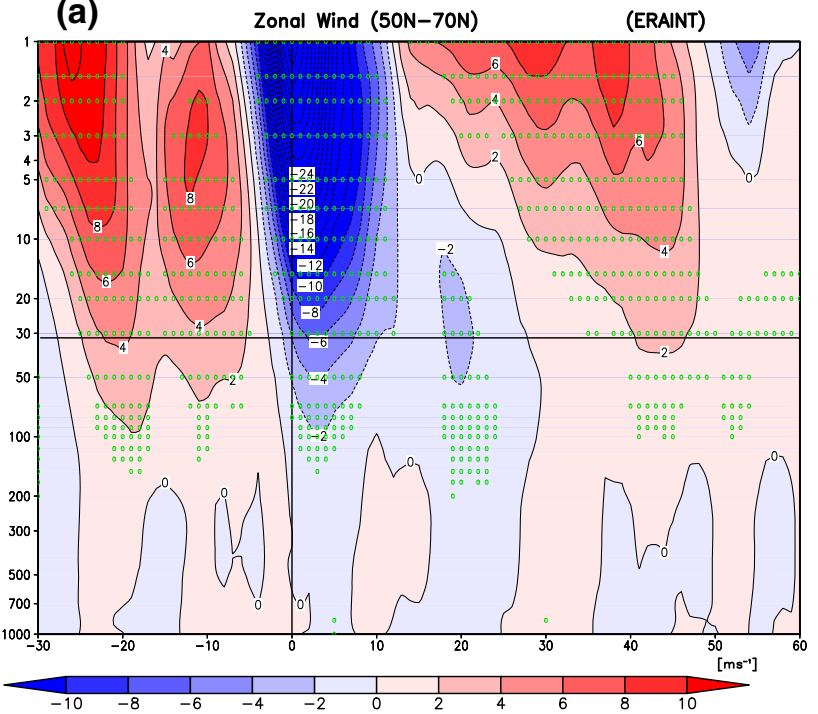

(c)

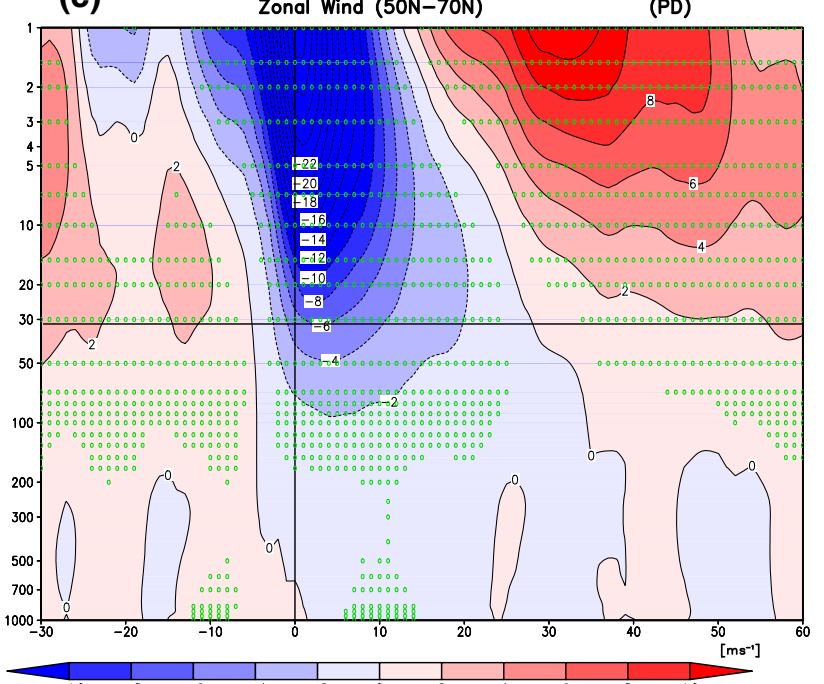

(b)

Zonal Temperature $(70 \mathrm{~N}-90 \mathrm{~N})$

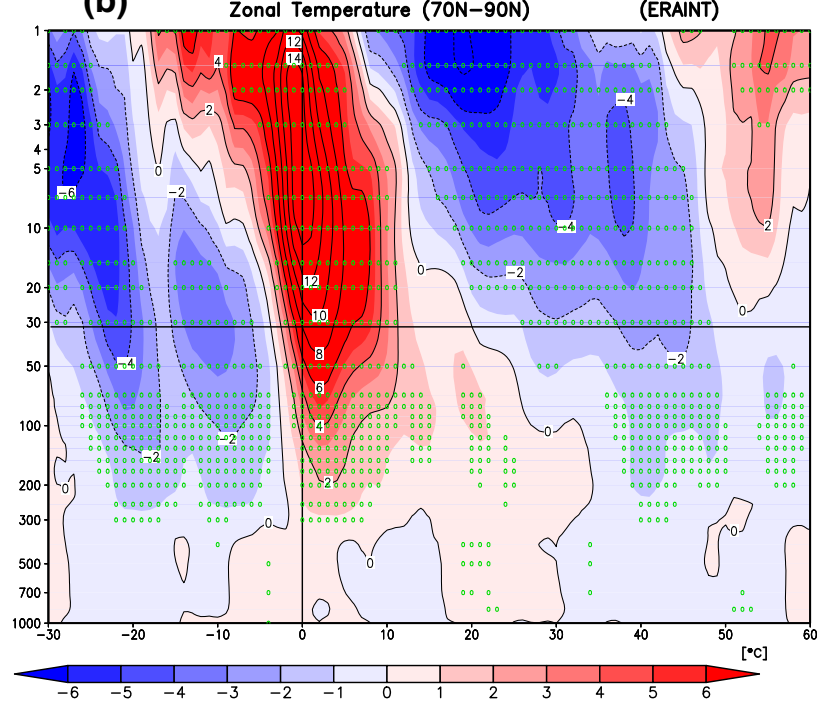

(d)

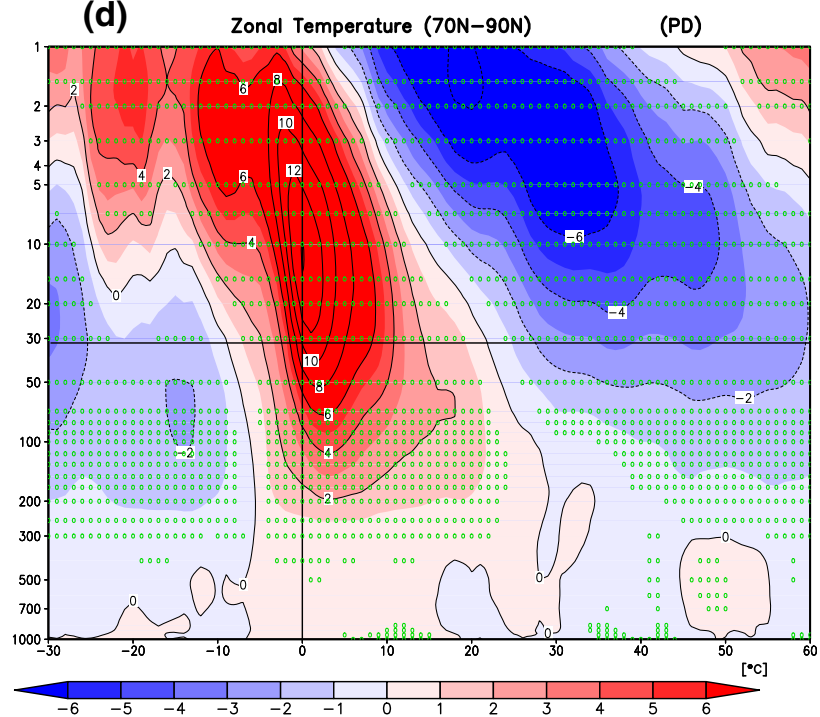

Figure 3. Left panels: Time series (days before and after the SSW) of the zonal-mean zonal wind (ms ${ }^{-1}$ ) averaged at $\left(50-70^{\circ} \mathrm{N}\right)$ and $10 \mathrm{hPa}$. Upper left column and lower left column depict for Era-Interim and PD respectively. Right panels: Time series (days before and after the SSW) of the zonal temperature $\left({ }^{\circ} \mathrm{C}\right.$ ) averaged at $\left(70-90^{\circ} \mathrm{N}\right)$ and $10 \mathrm{hPa}$ for EraInterim (upper right column) and PD (lower right column) respectively. ' 0 ' mark on the time axis denotes the actual date of the warming. Dots in each plots represent regions greater than $95 \%$ significance using Student- $t$ criterion.

levels from the surface $(1000 \mathrm{hPa})$ extends till 1 $\mathrm{hPa}$, is depicted in y-axis. Each variable in figures 4 and 5 are averaged over $50^{\circ}-70^{\circ} \mathrm{N}$. Regions over $95 \%$ statistical significance are shown as dots. EPflux is the main contributing factor to the vertical zonal flow (Albers and Birner 2014; Monier and Weare 2011) among the other factors like residual or gravity wave drag. Left column of figure 4 shows EP-flux divergence anomalies. EP-flux divergence anomalies is strongly negative in the stratosphere prior to the central date in displacement and split SSW of Era-Interim and PD (figure 4a-d). A negative EP-flux in split type (figure $4 \mathrm{~b}$ ) and (figure 4d) which has a larger magnitude and exist at a lower altitude than that in displacement type SSW. Moreover, in split type SSW, the negative EP-flux divergence in both simulation and observation begins earlier than displacement type. The negative EP-flux forcing is enhanced by more than $3 \mathrm{~ms}^{-1}$ per day for both types at approximately day $=0$. However, the EP-flux forcing anomaly of split type is greatest during the period between day $=-3$ and day $=+3$ and has a secondary peak at approximately day $=-7$ in observation and PD. In contrast, a single peak exists at day $=0$ for displacement type SSW is observed in Era-Interim 
(a) EP-FLUX DIVRGENCE (50-70N)

(ERANT)

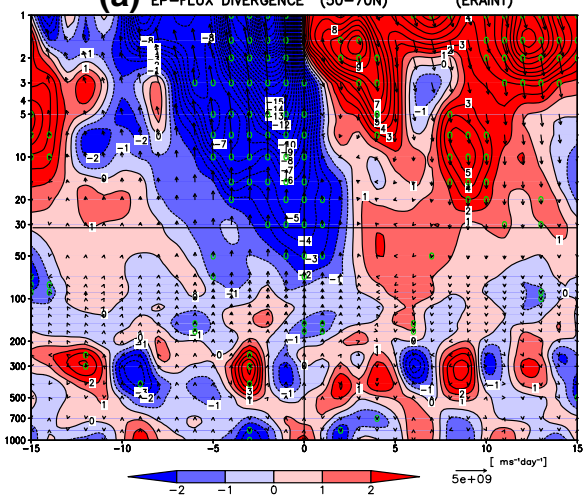

(b) EP-FLUX DNERGENCE (SPLIT) (50-7ON)

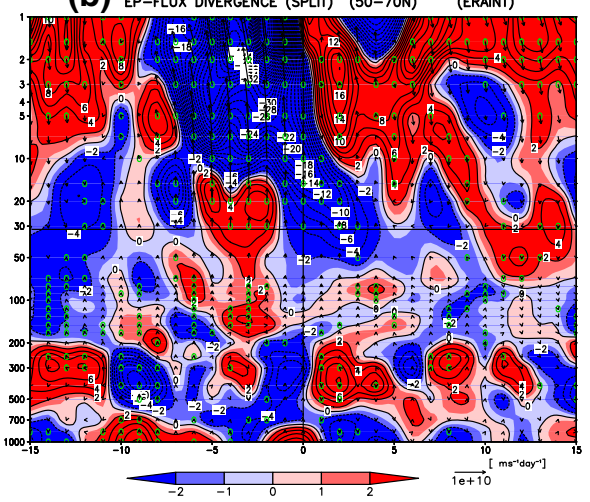

(c)

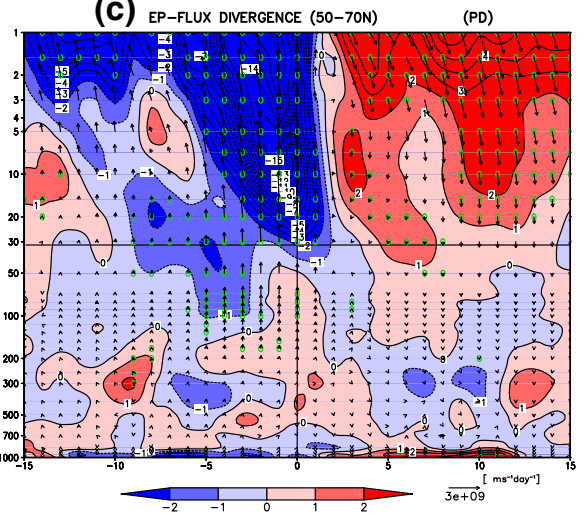

(d) EP-FLUX DIVERGENCE (SPLT) (50-7ON)

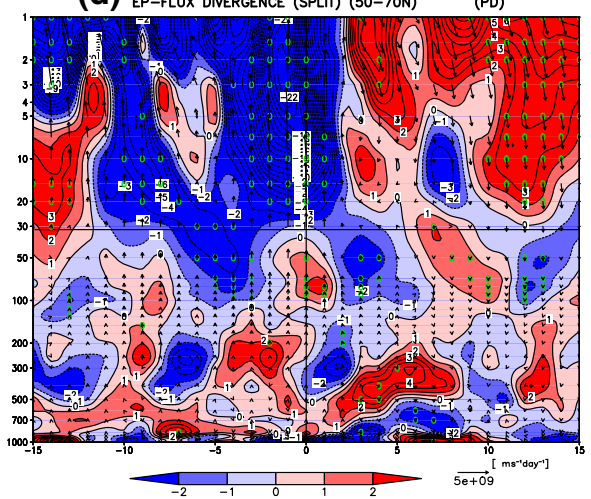

(e) ZONAL WIND TENDENCY (50-7ON)

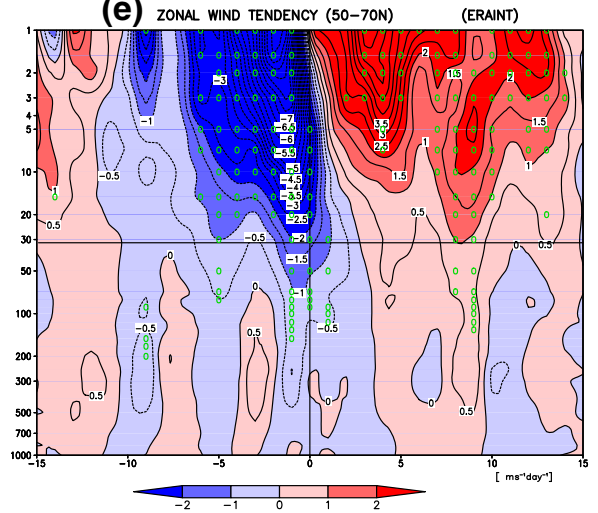

(f) ZONAL WIND TENDENCY (SPLT) (50-7ON)

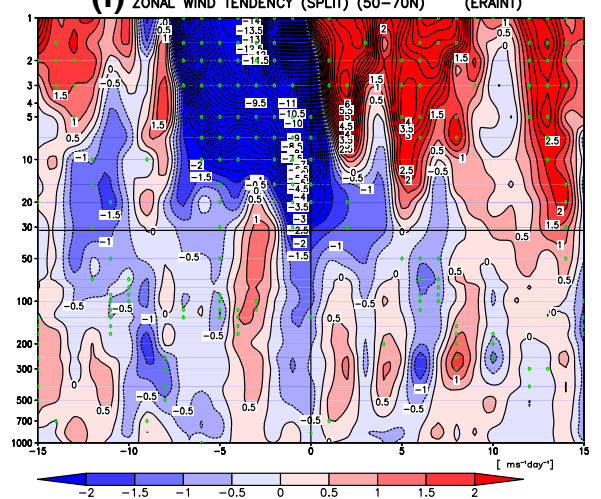

(g) ZONAL WIND TENOENCY (50-7ON)

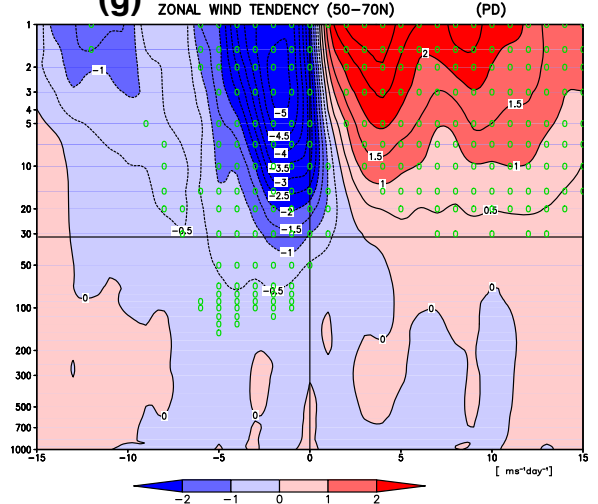

(h) ZONAL WIND TENDENCY (SPLIT) (50-7ON) (PD)

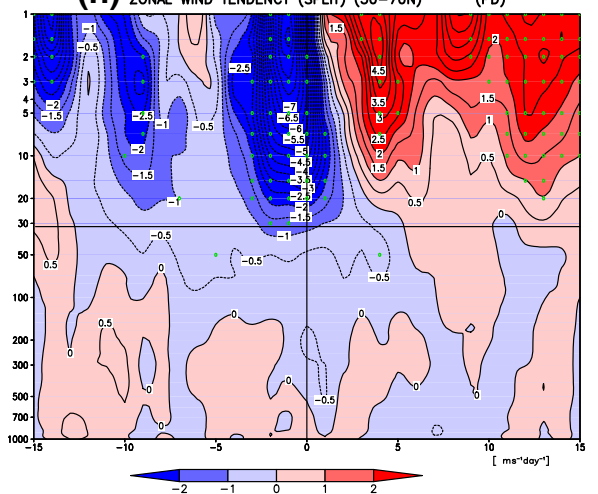

Figure 4. Time-height cross-section of the EP-flux divergence and the EP-flux vectors (left column), zonal wind tendency (right column) for displacement and split SSW events averaged over $50^{\circ}-70^{\circ} \mathrm{N}$ in Era-Interim and PD respectively. The horizontal axis shows 15 days before and after the actual date of the warming and vertical axis denotes the height in (hPa). Dots on each figure represent $95 \%$ statistical significance using Student $t$-test. Each variable is measured in ms ${ }^{-1} \mathrm{day}^{-1}$. 

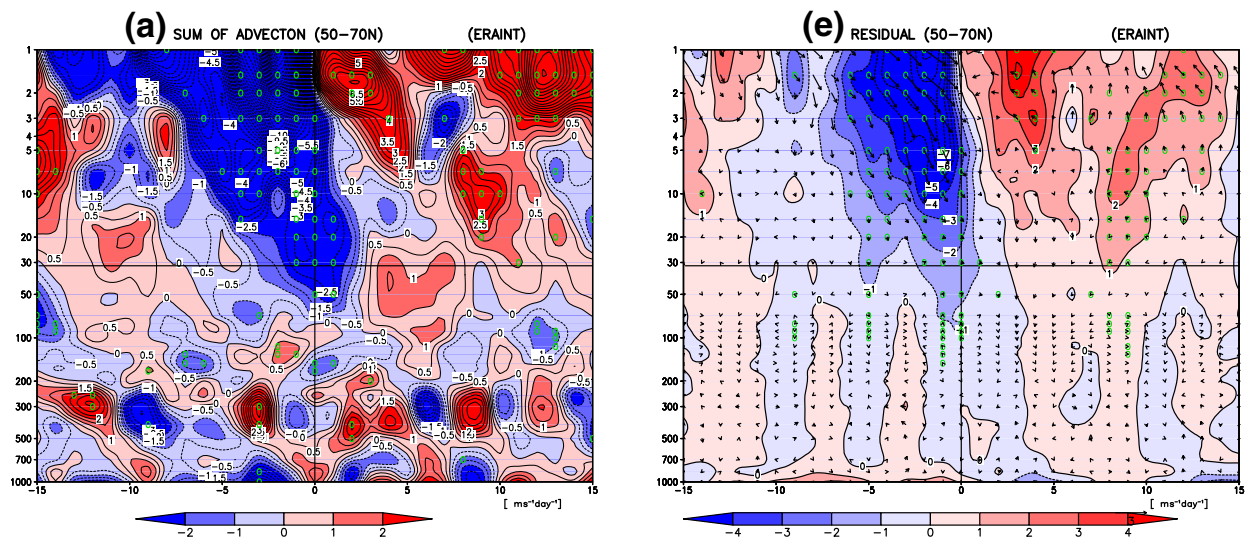

(b) SUM OF ADVECTION (SPLT) (50-7ON)

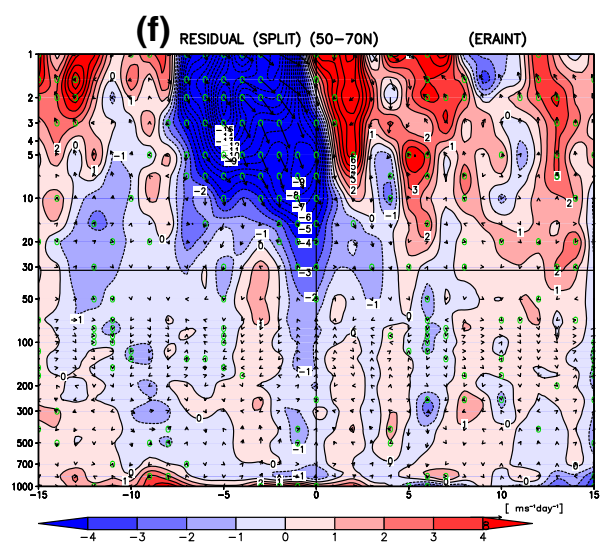

(c) SUM OF ADVECTION (50-70N)

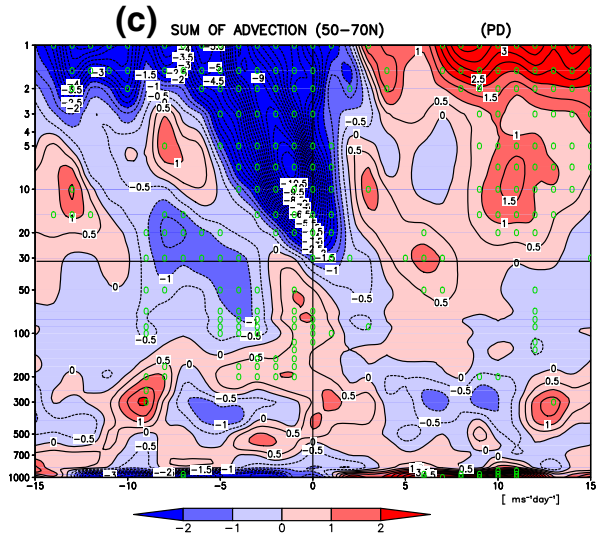

(g) RESIDUAL (50-70N)

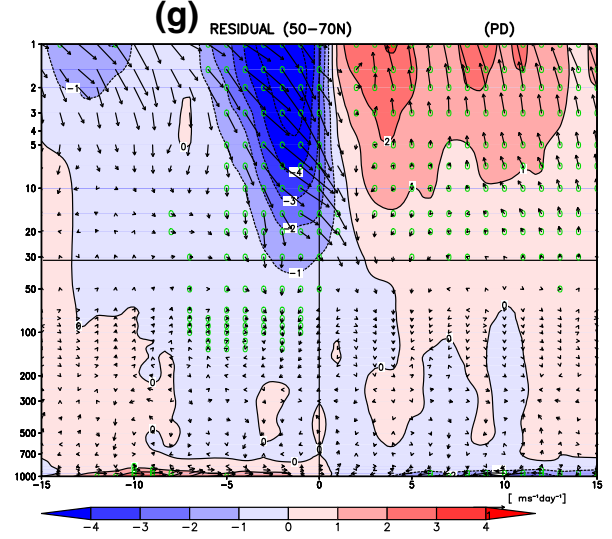

(d) SUM OF ADVECTION (SPLIT) (50-70N)

(PD)

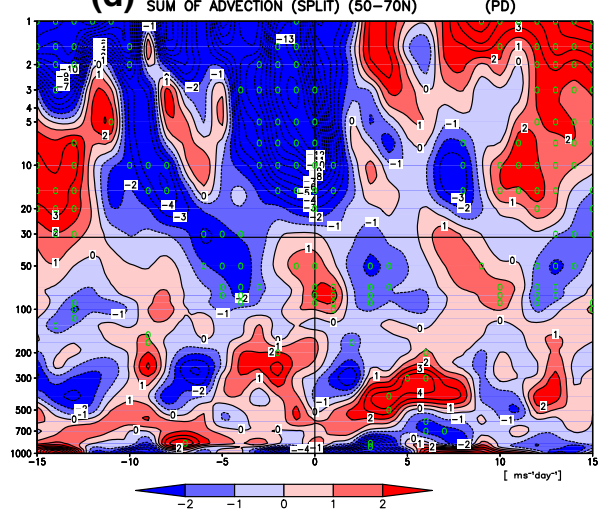

(h) RESIDUAL (SPLIT) (50-70N)

(PD)

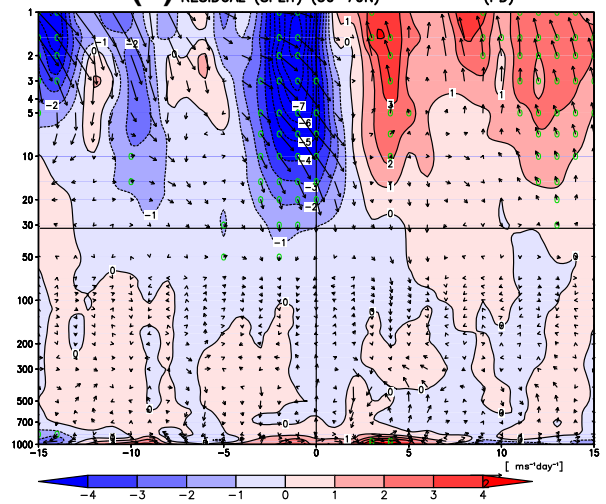

Figure 5. Same as figure 4, but here the left column shows sum of advection and right column depicts the residual term with the residual mean circulation vectors for displacement and split SSW events. 
and PD. EP-flux vector is a useful diagnostic tool for the net propagation of wave energy by planetary waves from a given region and height to another latitude. This flux vector of figure 4 (left column) is vertically dominated indicating upward propagation of planetary wave energy into the stratosphere from the troposphere during warming. The EP-flux vectors in split SSW with its maximum strength appears earlier in observation, i.e., few days before the key date than in the simulation. The flux vectors analyzed for the model simulation captures a similar pattern around the key date like observation in displacement type SSW. The zonal wind tendency shown in figure $4(\mathrm{e}-\mathrm{h})$ is a direct consequence of the EP-flux convergence and divergence discussed before. For instance, EP-flux convergence accelerates the wind in easterly direction (negative zonal wind tendency). Similar to the EP-flux, the wind tendency in split type (figure $4 \mathrm{f}$ and $\mathrm{h}$ ) has a larger magnitude (more than $2 \mathrm{~ms}^{-1}$ per day) than that in displacement type (figure 4e and $\mathrm{g}$ ). The secondary maximum is also observed in split type approximately on day $=-7$ in Era-Interim and well presented in PD. The sum of advection as shown in figure $5(\mathrm{a}-\mathrm{d})$ shows slightly smaller in magnitude compared to EP-flux (Monier and Weare 2011) except for split events in Era-Interim. The sum of advection (figure 5b) and EP-flux convergence (figure $4 \mathrm{~b}$ ) of split events in Era-Interim on day $=0$ produce similar pattern and approximately equal to $8 \mathrm{~ms}^{-1}$ per day in magnitude. In $\mathrm{PD}$, the advection term is less than EP-flux by $1 \mathrm{~ms}^{-1}$ per day on day $=0$. The advection term in split events like the EP-flux produces secondary maximum 5 days prior to final warming for both the datasets.

The residual term may be considered as gravity wave drag or other unresolved processes not included in EP-flux or friction and contributes significantly to the momentum budget. The influence of subgrid scale orography on the momentum of the atmosphere is represented by a combination of lower tropospheric drag due to the absorption and reflection of vertically propagating gravity waves generated by stably stratified flow over the subgrid scale orography. The gravity wave drag anomaly in all SSWs presented in figure $5(\mathrm{e}-\mathrm{h})$ has small positive values on day $=-15$ and negative values begin to develop from then onwards in the upper stratosphere. However, in split type events of Era-Interim, the negative anomaly begins earlier (figure 5f). Negative gravity wave forcing is enhanced in split type SSW compared to displacement type in the upper stratosphere for observation and PD. Similar to advection term, residual term have less value in terms of magnitude compared to EP-flux. In addition to gravity wave drag forcing considered as residual term, the resultant of the horizantal and vertical component of residual circulation in vector is plotted in figure $5(\mathrm{e}-\mathrm{h})$. The meridional and vertical components of the residual mean circulation have largest value and moving downward around the central date.

\section{Conclusions and discussion}

In this present study, composite analysis for 76 warming events from 300 model years of present day simulation is performed to investigate the residual mean circulation during the evolution of the major stratospheric sudden warming. The dynamical signatures that are obtained from the model were compared with the composites of 17 SSWs using Era-Interim datasets. The number of SSW/winter and its monthly distribution from model is underestimated in comparison with SSW number in Era-Interim. The efficiency of the algorithm used to identify the central date of each SSW was checked with the observed SSW from FUB. The number of SSW in Era-Interim (17 events) agrees well with FUB (18 events). Therefore, the model simulates smaller numbers of SSW and that might be explained due to permanent easterly winds in the tropics, i.e., missing QBO (Schimanke et al. 2013). We have further classified the SSW events in model as displacement (66 events) and split type (10 events) based on the stereographic projection of geopotential field values at $10 \mathrm{hPa}$. The number of displacement SSW (14 events) and split SSW (3 events) from EraInterim is also less when compared with Charlton and Polvani (2007) and may be due to the fact that our method is not algorithm based rather selected from the geopotential plots.

Studies (Bal et al. 2017; Kodera et al. 2014) over the years have suggested that the stratosphere is influenced by the troposphere during sudden stratospheric warming. However, there are studies that stratosphere not only receives waves, but also return energy back to the troposphere in the form of convective activity (Bal et al. 2017; Kodera et al. 2011). The convective activity is enhanced in the equatorial northern hemisphere and suppressed in the southern hemisphere during SSW was studied by Kodera et al. (2011) and Bal 
et al. (2017). The underlying mechanism for the convective activity taking place in the equatorial troposphere during SSW were explained by Kodera (2006), Kodera et al. (2011) and Bal et al. (2017). This link between the stratosphere and troposphere is the enhancement of vertical rising planetary waves. This is evident in our study that after the final warming day, the stratospheric easterly anomalies near the northern pole moves down to the troposphere, while the easterly anomalies in the upper stratosphere begin to decelerate and reverse towards the westerly circulation (figure 3a and c). Similarly, the positive zonal temperature anomalies around the pole descend to the troposphere after the warming, whereas in stratosphere the zonal temperature anomalies begin to cool and return to its normal state (figure $3 \mathrm{~b}$ and d). Recently Bal et al. (2017) have reported that the impact on the troposphere is more robust in split vortex type than displacement SSW. So, in this present research effort, we have verified the residual mean circulation anomalies in different types of sudden stratospheric warming for PD and compared with observation. Our study confirms that various processes (divergence of EP-flux or planetary wave forcing, advection of zonal-mean zonal momentum and the residual term or gravity wave drag) forcing the zonal-mean zonal momentum tendency is stronger for split SSW than for displacement SSW. It is also noticed that the occurrences of sudden stratospheric warming and the strong downward motion after the key date is produced primarily by the planetary wave forcing (EP-flux divergence). The other forcings like residual term (gravity wave) or advection term executes a smaller contribution to the residual mean circulation than EP-flux divergence. As the number of $\mathrm{SSW} /$ winter simulated is less in model, the realistic model should be simulated to check the underlying physics minutely. Therefore, future studies should include more strong SSW as there are limited SSW in observation.

\section{Acknowledgements}

We thank Dr Markus Kunze for providing us the routine to calculate the EP-flux parameters. We are thankful to Dr Rajendran Kavirajan, the editor and to the anonymous reviewers for useful and helpful comments that improved this manuscript substantially. Sourabh Bal was supported by the Erasmus Mundus (EMINTE) post-doctoral scholarship for nine months at FU, Berlin.

\section{References}

Albers J R and Birner T 2014 Vortex preconditioning due to planetary and gravity waves prior to sudden stratospheric warmings; J. Atmos. Sci. 71(11) 4028-4054.

Andrews D G, Holton J R and Leovy C B 1987 Middle atmosphere dynamics; 40, Academic Press.

Bal S, Schimanke S, Spangehl T and Cubasch U 2017 Variable influence on the equatorial troposphere associated with SSW using Era-Interim; J. Earth Syst. Sci. 126(2) 19.

Baldwin M P and Dunkerton T J 2001 Stratospheric harbingers of anomalous weather regimes; Science 294(5542) 581-584.

Butler A H, Seidel D J, Hardiman S C, Butchart N, Birner T and Match A 2015 Defining sudden stratospheric warmings; Bull. Am. Meteor. Soc. 96(11) 1913-1928.

Charlton A J and Polvani L M 2007 A new look at stratospheric sudden warmings. Part I: Climatology and modeling benchmarks; J. Clim. 20(3) 449-469.

Charney J G and Drazin P G 1961 Propagation of planetaryscale disturbances from the lower into the upper atmosphere; J. Geophys. Res. 66(1) 83-109.

Dee D, Uppala S, Simmons A, Berrisford P, Poli P, Kobayashi S, Andrae U, Balmaseda M, Balsamo G and Bauer P et al. 2011 The Era-Interim reanalysis: Configuration and performance of the data assimilation system; Quart. J. Roy. Meteorol. Soc. 137(656) 553-597.

Gómez-Escolar M, Calvo N, Barriopedro D and Fueglistaler S 2014 Tropical response to stratospheric sudden warmings and its modulation by the QBO; J. Geophys. Res. Atmos. 119(12) 7382-7395.

Harada Y, Goto A, Hasegawa H, Fujikawa N, Naoe H and Hirooka T 2010 A major stratospheric sudden warming event in January 2009; J. Atmos. Sci. 67(6) 2052 2069.

Huebener H, Cubasch U, Langematz U, Spangehl T, Niehörster F, Fast I and Kunze M 2007 Ensemble climate simulations using a fully coupled ocean-tropospherestratosphere general circulation model; Phil. Trans. A. Math. Phys. Eng. Sci. 365(1857) 2089-2101.

Kodera K (2006) Influence of stratospheric sudden warming on the equatorial troposphere; Geophys. Res. Lett. 33(6) L06804, https://doi.org/10.1029/2005GL024510.

Kodera K, Eguchi N, Lee J N, Kuroda Y and Yukimoto S 2011 Sudden changes in the tropical stratospheric and tropospheric circulation during January 2009; J. Meteorol. Soc. Japan 89(3) 283-290.

Kodera K, Funatsu B, Claud C and Eguchi N 2014 The role of convective overshooting clouds in tropical stratospheretroposphere dynamical coupling; Atmos. Chem. Phys. Discuss. 14(16) 23,745-23,761.

Kuroda Y 2008 Effect of stratospheric sudden warming and vortex intensification on the tropospheric climate; J. Geophys. Res. Atmos. 113(D15) D15110, https://doi.org/10. 1029/2007JD00955.

Labitzke K 1981 Stratospheric-mesospheric midwinter disturbances: A summary of observed characteristics; J. Geophys. Res. Oceans 86(C10) 9665-9678.

Labitzke K and Naujokat B 2000 The lower arctic stratosphere in winter since 1952; Sparc Newslett. 15 $11-14$. 
Legutke S, Voss R, Klimarechenzentrum D and Betreuungsgruppe M 1999 The Hamburg atmosphere-ocean coupled circulation model ECHO-G, vol. 18, DKRZ.

Limpasuvan V, Richter J H, Orsolini Y J, Stordal F and Kvissel O K 2012 The roles of planetary and gravity waves during a major stratospheric sudden warming as characterized in WACCM; J. Atmos. Sol-Terr. Phys. 78 8498.

Matsuno T 1971 A dynamical model of the stratospheric sudden warming; J. Atmos. Sci. 28(8) 1479-1494.

Mitchell D M, Charlton-Perez A J and Gray L J 2011 Characterizing the variability and extremes of the stratospheric polar vortices using $2 \mathrm{~d}$ moment analysis; J. Atmos. Sci. 68(6) 1194-1213.

Monier E and Weare B C 2011 Climatology and trends in the forcing of the stratospheric zonal-mean flow; Atmos. Chem. Phys. 11(24) 12,751-12,771.

Ryoo J M and Chun H Y 2005 Stratospheric major sudden warmings revealed in NCEP reanalysis data for 41 years (1958-1999); J. Korean. Meteorol. Soc. 41 173-190.

Scherhag R 1952 Die explosionsartigen stratosphärenerwä rmungen des spätwinters 1951/52; Berichte des deutschen Wetterdienstes in der US-Zone 6(38) 51-63.
Schimanke S, Krper J, Spangehl T and Cubasch U 2011 Multi-decadal variability of sudden stratospheric warmings in an AOGCM; Geophys. Res. Lett. 38(1), https:// doi.org/10.1029/2010GL045756, 101801.

Schimanke S, Spangehl T, Huebener H and Cubasch U 2013 Variability and trends of major stratospheric warmings in simulations under constant and increasing GHG concentrations; Clim. Dyn. 40(7-8) 1733-1747.

Schoeberl M R 1978 Stratospheric warmings: Observations and theory; Rev. Geophys. 16(4) 521-538.

Spangehl T, Cubasch U, Raible C, Schimanke S, Körper J and Hofer D 2010 Transient climate simulations from the maunder minimum to present day: Role of the stratosphere; J. Geophys. Res. Atmos. 115(D1) D00110, https://doi.org/10.1029/2009JD012358.

Taguchi M 2011 Latitudinal extension of cooling and upwelling signals associated with stratospheric sudden warmings; J. Meteorol. Soc. Japan 89(5) 571-580.

de la Torre L, Garcia R R, Barriopedro D and Chandran A 2012 Climatology and characteristics of stratospheric sudden warmings in the whole atmosphere community climate model; J. Geophys. Res. Atmos. 117(D4) D04110, https://doi.org/10.1029/2011JD016840.

Corresponding editor: K RAJENDRAN 